\title{
ESTÁGIO DOCÊNCIA: DIFICULDADES ENCONTRADAS PELOS ESTAGIÁRIOS NA DISCIPLINA DE CÁLCULO I
}

\section{TEACHING INTERNSHIP: DIFFICULTIES FOUND BY TRAINEES IN THE DISCIPLINE OF CALCULUS I}

\author{
Tiely Virgínio da Hora Lima ${ }^{1}$
}

UFRN

\section{Liliane dos Santos Gutierre ${ }^{2}$}

UFRN

\section{Resumo}

Nesse artigo, apresentaremos parte da nossa pesquisa, que se iniciou em agosto de 2018, quando fomos contemplados com uma bolsa de Iniciação Científica (IC) e desde então passamos a frequentar o Grupo de Estudos e Pesquisas em História da Educação Matemática (GPEP) da Universidade Federal do Rio Grande do Norte (UFRN). O objetivo geral da pesquisa e não desse artigo é analisar como se deu o estágio à docência, no período de 2008 a 2018, de estudantes de Pós-Graduação que participaram do Programa de Assistência à Docência na Graduação (PADG), atuando na disciplina de Cálculo I e suas variações, nos cursos de Graduação da UFRN, no campus Natal. A pesquisa se dará por meio de uma pesquisa documental (SÁ-SILVA, 2009). Ela ainda está em andamento, entretanto, já iniciamos nossa coleta de dados, por meio do site www.sigaa.ufrn.br e visualizamos que a UFRN, campus Natal, tem 84 (oitenta e quatro) cursos na modalidade presencial e 10 na modalidade a distância. Desses, há 37 (trinta e sete) da modalidade presencial e 4 (quatro) da modalidade à distância que possuem, em sua estrutura curricular, uma disciplina cuja ementa possui derivação e integração de funções reais de uma variável real. Temos na referida instituição cadastrada 127 cursos de Pós-Graduação na modalidade stricto sensu, destes cursos buscamos os que tiveram os estagiários ministrando a disciplina de Cálculo I para poder analisar seus relatórios. Por fim, consideramos importante divulgar esses resultados, mesmo que parciais. Eles foram apresentados no II Seminário do GPEP, realizado no campus IV da Universidade Federal da Paraíba (UFPB), em Rio Tinto/PB. Vale lembrar que, nesse artigo, também fazemos uma apresentação do nosso referencial teórico, uma vez que, em outra oportunidade, apresentaremos os resultados finais da pesquisa.

Palavras-chave: Ensino; Cálculo; Estágio Docência; PADG.

\begin{abstract}
In this article, we will present part of our research, which began in August 2018, when we were awarded a scholarship in Scientific Initiation and since then we have been attending the Grupo de Estudos e Pesquisas em História da Educação Matemática (GPEP) [Group of Studies and Research in History of Mathematics Education] of the Universidade Federal do Rio Grande do Norte (UFRN). [Federal University of the Rio
\end{abstract}

${ }^{1}$ tielyvirginio@gmail.com.

2 lilianegutierre@gmail.com. 
Grande do Norte]. The general objective of the research and not of this article is to analyze how the stage of teaching internship took place, in the period from 2008 to 2018, of postgraduate students who participated in the Programa de Assistência à Docência na Graduação (PADG) [Program of Assistance to Teaching in Graduation], acting in the discipline of Calculus I and its variations, in the postgraduate courses of UFRN, at the Natal campus. The research will be done through a documentary research (SÁ-SILVA, 2009). The research is still ongoing, however, we have already started our data collection, through the site www.sigaa.ufrn.br and we visualize that (UFRN), Natal campus, has 84 (eighty-four) courses in the In-person mode and 10 in the distance mode. Of these, there are 37 (thirty-seven) of the face-to-face modality and 4 (four) of the distance modality they have, in their curricular structure, a discipline whose menu has derivation and integration of real functions of a real variable. We have 127 registered postgraduate courses in the stricto sensu modality, of these courses we looked for those that had the trainees ministering the discipline of Calculus I to be able to analyze their reports. Finally, we consider it important to disclose these results, even if partial. They were presented at the II GPEP Seminar, held on campus IV of the Universidade Federal da Paraíba (UFPB) [Federal University of Paraíba], in Rio Tinto/PB. It is worth remembering that in this article we also present our theoretical framework, since, in another opportunity, we will present the final results of the research.

Keywords: Teaching; Calculus; Teaching Internship; PADG.

\section{Introdução}

No atual cenário da educação, percebe-se que existem dificuldades em sistematizar métodos de ensino, quando se tratam de disciplinas em Cursos de Graduação cuja ementa contenha conteúdos relacionados à derivação e integração de funções reais de uma variável real. Os desafios e dificuldades do ensino da Matemática vêm desde o Ensino Básico e se evidenciam nos anos inicias da graduação, conforme nos apontam estudiosos da área, quando nos dizem que não é difícil ouvir de professores e pesquisadores que "os alunos estão ingressando nas universidades cada vez mais despreparados" (MASOLA,2016, p. 64).

Desse modo, entendemos ser fundamental estudar sobre as práticas e os métodos de ensino abordados na Graduação, principalmente, nas disciplinas, como a de Cálculo $\mathrm{I}^{3}$, que normalmente, é oferecida no primeiro semestre dos cursos, aos ditos calouros. Nossa motivação em desenvolver essa pesquisa partiu de um convite da professora líder do Grupo de Estudos e Pesquisas em História da Educação Matemática (GPEP) da

\footnotetext{
${ }^{3}$ Neste texto, nos remeteremos ao nome Cálculo I a toda e qualquer disciplina que tenha em sua ementa o conteúdo de limites, derivadas e integrais de função real de variável real.
} 
Universidade Federal do Rio Grande do Norte (UFRN), quando, em agosto de 2018, fomos contemplados com uma bolsa de Iniciação Científica (IC) e começamos a frequentar o referido grupo.

Posto isto, objetivo geral da pesquisa é analisar como se deu o estágio à docência, no período de 2008 a 2018, de estudantes de Pós-Graduação que participaram do Programa de Assistência à Docência na Graduação (PADG), atuando na disciplina de Cálculo I, nos cursos de graduação da UFRN, no campus Natal. Na expectativa de atingir esse objetivo, lançamos mão da pesquisa documental, que será apresentada a seguir. Vale dizer que, nesse artigo, apresentaremos resultados parciais, pois a pesquisa está em andamento, mas, apresentaremos, também, o referencial teórico que estamos lançando mão para cumprirmos o objetivo proposto.

\section{Metodologia da pesquisa}

Em nossa pesquisa iremos nos valer dos conceitos da pesquisa documental para podermos analisar cada relatório (documento) do Estágio Docência dos pós-graduandos, verificando, assim, o que nos diz os números que cada relatório traz, além dos conteúdos presentes, a partir da perspectiva dos estagiários da docência assistida, entre outros aspectos.

Segundo Bravo (1991), documentos são as realizações produzidas pelo homem que se mostram como indícios de sua ação, podendo revelar suas ideias, opiniões e formas de atuar e viver. Desta forma, os documentos não se restringem apenas aos escritos, podendo ser ainda os numéricos ou estatísticos, os de reprodução de som e imagem e os documentos-objeto. (SILVA et al, 2009).

De acordo com Sá-Silva et al (2009, p. 4-5), a pesquisa documental é definida como um procedimento que se utiliza de métodos e técnicas para apreensão, compreensão e análise de documentos dos mais variados tipos. Outra definição é dada por Pádua (1997, p. 62) que diz "é aquela realizada a partir de documentos, contemporâneos ou retrospectivos, considerados cientificamente autênticos (não fraudados)". 
A análise de conteúdo é para Bravo (1991) a técnica mais elaborada e de maior prestígio no campo da observação documental, a constituir um meio para estudo das comunicações entre os homens, enfatizando assim o conteúdo de suas mensagens.

Entender as mensagens que os relatórios dos estagiários mostram quanto à metodologia de ensino utilizada e, além disso, a formação que o estagiário está obtendo é primordial para o estudo em questão, tendo em vista que estes relatórios ainda não foram analisados por outrem (com esse objetivo), de modo que faremos uma análise de forma criteriosa de cada relatório. Gil (2008) nos mostra o que seria a pesquisa documental e como diferenciá-la da pesquisa bibliográfica,

\begin{abstract}
A pesquisa documental assemelha-se muito à pesquisa bibliográfica. A única diferença entre ambas está na natureza das fontes. Enquanto a pesquisa bibliográfica se utiliza fundamentalmente das contribuições dos diversos autores sobre determinado assunto, a pesquisa documental vale-se de materiais que não receberam ainda um tratamento analítico, ou que ainda podem ser reelaborados de acordo com os objetivos da pesquisa. (GIL 2008, p. 51).
\end{abstract}

Sá-Silva (et al,2009, p. 6, apud OLIVEIRA, 2007, p. 70) chama a atenção para o fato de que "na pesquisa documental, o trabalho do pesquisador(a) requer uma análise mais cuidadosa, visto que os documentos não passaram antes por nenhum tratamento científico", ideia que corrobora com a de Gil (2008).

De acordo com Silva (et al, 2009, p. 4559), é nessa etapa que os documentos são estudados e analisados de forma minuciosa. O pesquisador descreve e interpreta $\mathrm{o}$ conteúdo das mensagens, buscando dar respostas à problemática que motivou a pesquisa e, assim, corrobora com a produção de conhecimento teórico relevante.

Inicialmente, pesquisamos e vimos que tínhamos 84 cursos de graduação na modalidade presencial e 10 na modalidade à distância que fazem parte do campus Natal da UFRN. Em seguida, selecionamos cursos que possuem em sua grade curricular a disciplina de Cálculo I e suas variações, ou seja, que abrangem o conteúdo de derivação e integração de funções reais de uma variável real. Essa seleção foi realizada pelo site do SIGAA, na aba Graduação, que pode ser acessado por meio do link https://sigaa.ufrn.br/sigaa/public/curso/lista.jsf?nivel=G\&aba=p-graduacao.

Tendo em vista que já foram selecionados os cursos que tinham a disciplina de Cálculo I, ou disciplinas equivalentes, buscamos, posteriormente, os cursos de Pós 
Tiely Virgíio da Hora Lima, Liliane dos Santos Gutierre

Estágio Docência: dificuldades encontradas pelos estagiários na disciplina de Cálculo I

Graduação que tiveram estagiários da docência assistida nessas disciplinas. Feito isso, selecionamos os relatórios dos referidos estagiários, no período de 2008 a 2018. A escolha desse período se deve ao fato do PADG existir a partir de 2008. Para acessarmos os relatórios, contamos com a ajuda da professora líder do GPEP, que tem acesso a área de stricto sensu no Sistema Integrado de Gestão de Atividades Acadêmicas (SIGAA). A fonte essencial para obtermos essas informações é por meio do SIGAA.

Posto isto, enfatizamos que "pesquisas elaboradas a partir de documentos são importantes não porque respondem definitivamente a um problema, mas porque proporcionam melhor visão desse problema ou, então, hipóteses que conduzem à sua verificação por outros meios.” (GIL, 2002, p. 47). Entender a fala de Gil (2002) nos auxilia no fato de que podemos não apenas achar o que procuramos nos relatórios, mas compreender o que acontece, em especial, nas disciplinas de Cálculo I, no contexto da UFRN.

Utilizaremos os meios necessários e suficientes para obtenção dos dados, para que esta pesquisa possa contribuir para a sociedade de modo que haja mudanças reais e significativas no que se refere a aprendizagem do aluno da Graduação.

\section{Sobre o Estágio à Docência na UFRN}

Os Programas de Pós-Graduação vêm sendo cada vez mais visados pelos ingressantes dos cursos de Graduação em todo o Brasil. Sabemos que em 1965, foi criado o parecer $n^{\circ}$ 977, que institui o Programa de Pós-Graduação no Brasil e atribui uma nova característica às universidades Brasileiras. O "pai" da Pós-Graduação foi o Newton Sucupira, que proporcionou, com este parecer, contribuições relevantes à educação, pesquisa, cultura e ao aperfeiçoamento profissional (BOAVENTURA, 2009). No Brasil, a Pós-Graduação é dividida em strictu sensu e lato sensu, abrangendo, respectivamente, mestrado e doutorado, especialização e aperfeiçoamento.

No contexto da UFRN, verificamos um grande interesse a nível nacional pelos cursos de mestrado e doutorado oferecidos pela instituição. Atualmente, temos, nessa instituição, 127 cursos Stricto Sensu e 63 cursos Lato Sensu, conforme consta no SIGAA. Vale dizer que o Portal da UFRN nos mostra que o primeiro curso de Pós- 
Graduação foi o que hoje em dia é conhecido por Programa de Pós-Graduação em Saúde Coletiva. Sobre isso, a seguir, apresentamos um breve relato da história da criação desse curso:

O atual Programa de Pós-Graduação em Saúde Coletiva da UFRN iniciou suas atividades acadêmicas em fevereiro de 1978, sob a denominação de Curso de Mestrado em Odontologia Social, tendo sido aprovado pelo Colegiado Superior de Ensino, Pesquisa e Extensão da UFRN-CONSEPE, através da Resolução 60/77, de 03 de junho de 1977, se constituindo no primeiro curso de Pós-Graduação "stricto sensu" desta Universidade. (UFRN, (C) 2006-2019).

Em 1999, com o propósito de atender ao aperfeiçoamento docente dos mestrandos (as) e doutorandos (as) foi criada a Resolução no 100/99-CONSEPE, em 05 de outubro, na qual dá início ao Programa de Estágio a Docência na Graduação, entretanto, foi revogada pela Resolução n ${ }^{\circ} 063$ de 2010 , que tem por objetivos:

I - contribuir na formação para a docência de alunos de PósGraduação em nível de mestrado e doutorado por meio de atividades acadêmicas na Graduação; II - contribuir para a melhoria da qualidade de ensino nos Cursos de Graduação; III - contribuir para a articulação entre Graduação e Pós-Graduação. (UFRN, 2010, p. 2).

A participação no PADG da UFRN é obrigatória para os estudantes Bolsistas de Demanda Social e para aqueles cujo regimento do Programa deles exija. Na Resolução $n^{\circ}$ 063/2010 - CONSEPE, e em seu artigo sexto, consta que o estudante tem que concluir o Curso de Iniciação à Docência (CID) ou uma disciplina de cunho didático e pedagógico (MAGALHÃES et al, 2016, p.570, apud, UFRN, 2010, p. 2) para poder estagiar. A importância deste estágio e, principalmente, a conclusão do CID ou da disciplina, faz com que os estagiários confrontem suas expectativas sobre a prática docente, como expõem Magalhães et al, a partir do que diz Pimenta e Lima (2006),

Pimenta e Lima (2006) advogam que a formação para a profissão docente tem, indubitavelmente, uma dimensão prática, sendo comum uma aprendizagem para a docência marcada pela observação, imitação, reprodução e, mais raramente, pela reelaboração da prática habitual em processos de estágio. Contudo, esta perspectiva de formação docente nascida apenas da observação de modelos parece insuficiente, na medida em que o aluno nem sempre dispõe de 
elementos para a ponderação crítica das práticas pedagógicas observadas durante o estágio. (MAGALHÃES et al, 2016, p.567).

Sob a perspectiva de qualificar mais e mais o professor em sua formação iniciada e continuada, faz-se necessário um investimento de caráter contínuo, em todos os aspectos. Os estudos em torno deste tema vêm se destacando em toda a sociedade, pois se vê a indiscutível necessidade de formação de professores, sejam os de ensino básico ou de ensino superior.

Para Imbernón (2010), a formação docente vai muito além do modelo que se tem hoje. Esse autor nos diz que ainda há muita formação e pouca mudança, isso se deve ao fato de predominarem políticas e formadores que praticam com afinco uma formação transmissora e uniforme, muitas vezes, caracterizada por uma teoria descontextualizada, válida para todos sem diferenciação, que não abrange os problemas práticos e reais, sendo fundamentada em um educador ideal que não existe.

Buscar mudanças no ensino é uma tarefa diária para todos que se empenham em formar professores. Segundo Freire (2014), na formação permanente dos professores, o principal momento é o da reflexão crítica sobre a prática, por meio do pensamento crítico de hoje ou de ontem podemos então melhorar a próxima prática.

É do nosso entendimento que a universidade, em seu caráter formativo, traz aos alunos um pensar crítico, durante a Graduação e que se acentua à medida que há o ingresso nos Programas de Pós-Graduação. Assim, podemos dizer que é de fundamental importância o PADG na formação desses novos pesquisadores, uma vez que podem atuar no ensino superior na condição de professores.

Quando se trata da disciplina de Cálculo I, observamos, por meio dos relatórios que iniciamos nossa análise, um cenário preocupante, pois, muitas vezes, há uma resistência dos professores efetivos, mais antigos, em se pensar os métodos e práticas dos que estão em formação inicial. Essas discussões devem ser prioridades a toda comunidade acadêmica, de modo que as dificuldades dos iniciantes dos cursos de licenciatura e bacharelado sejam sanadas, para que o desenvolvimento do estudante seja completo em todos os aspectos que a universidade proporciona.

Para tanto, vemos que no atual cenário da educação, existem dificuldades em sistematizar métodos de ensino, quando se tratam de disciplinas em cursos de 
Graduação cuja ementa contenha conteúdos relacionados à derivação e integração de funções reais de uma variável real (SILVA, 2017).

Segundo Nasser (2009), esse cenário é mundial, ou seja, em todos os cursos que possuem conteúdos matemáticos no currículo, tais como derivação e integração de funções reais de uma variável real, ocorre um elevado número de reprovação e não se restringe apenas aos estudantes brasileiros.

Podemos aqui elencar algumas dificuldades que estes estagiários encontram tais como: (i) a bagagem de conhecimentos dos alunos ingressantes; (ii) o interesse desses pelos cursos no qual ingressaram, tendo em vista que alguns alunos "entram" em cursos de baixa concorrência, apenas para ingressar na instituição pública; (iii) a resistência dos alunos às novas formas de ensino. Manter o modelo tradicional ou inovar na maneira de se ensinar é ou pode chegar a ser uma barreira para estes estagiários.

Os relatórios dos estágios nos ajudarão a entender como está sendo parte da formação destes iniciantes à docência, ou seja, como se dá a relação orientadorestagiário e estagiário-aluno, assim como o andamento da disciplina e os resultados obtidos. A análise dos índices de não aprovação, aprovação e trancamentos servirão para termos um entendimento de como está o ensino desta disciplina na UFRN, tendo em vista que já existem estudos que mostram os mesmos índices em outras universidades, como o estudo de Alvarenga, Dorr e Vieira (2016), que nos trás números de três instituições de ensino superior no centro-oeste brasileiro.

Para o meio acadêmico e para a sociedade entender a formação docente em seu processo e consequências, entendemos que nossa pesquisa nos fará ver com um olhar diferenciado o ser professor e como esta profissão deve ser cuidada por todos, para que o docente em sua formação inicial e continuada seja valorizado e, principalmente, pelos órgãos políticos que fazem as legislações que regem esta prática, que vai além do conteúdo transmitido em sala.

Nesse sentido, fizemos nesta pesquisa o levantamento de todos os cursos de Graduação da UFRN, campus Natal, que tenham em sua estrutura curricular a disciplina de Cálculo I e, como resultado parcial, identificamos o seguinte: são oferecidos as disciplinas de Cálculo I para 3 cursos da área de Biociências e para 34 cursos da área Tecnológica na modalidade presencial, já para a modalidade a distância, temos a oferta para 3 da área Tecnológica e 1 curso na área de Ciências Sociais Aplicada. Verificamos 
Tiely Virgíio da Hora Lima, Liliane dos Santos Gutierre

Estágio Docência: dificuldades encontradas pelos estagiários na disciplina de Cálculo I

quais possuem estudantes de Pós-Graduação realizando estágio. Após, analisamos seus relatórios, sob a perspectiva de investigar a metodologia aplicada pelos estagiários. Nossa pesquisa continua e brevemente apresentaremos nossas conclusões finais, atendendo ao objetivo proposto.

\section{Considerações finais}

Era nosso desejo divulgarmos, neste renomado periódico, parte dessa pesquisa, que, também, foi apresentada no II Seminário do GPEP, que aconteceu em Rio Tinto, no estado da Paraíba, na Universidade Federal da Paraíba. Esse evento nos proporcionou conhecimentos e contribuições. Estamos cientes que ainda temos muito a pesquisar, mas, com as considerações realizadas por parte dos professores que mediaram o Grupo de Trabalho (GT), durante o referido Seminário, junto a nossa dedicação, enquanto estudantes da iniciação científica, obteremos um trabalho significativo para a sociedade e, em breve, publicaremos os resultados finais.

Entendemos também ser importante considerar que, ao frequentarmos as reuniões do GPEP, assim como, quando participamos de um encontro local, regional, nacional ou internacional, cujos estudos se voltam para a área do grupo de estudos e pesquisas que tivemos a oportunidade de frequentar, nos "alimenta", permitindo dialogarmos com autores da área, a fim de realizarmos o estado da arte da nossa pesquisa e, de fato, contribuirmos com as pesquisas do GPEP, pois a nossa temática é voltada para a formação de professores, temática essa que o grupo também pesquisa.

\section{Referências}

ALVARENGA, K. B. DORR, R. C. VIEIRA, V. D. O ensino e a aprendizagem de cálculo diferencial e integral: características e interseções no centro-oeste Brasileiro. REBES - Rev. Brasileira de Ensino Superior, 2(4): 46-57, out.-dez. 2016 ISSN 2447-3944.

BOAVENTURA, EM. A construção da universidade baiana: objetivos, missões e afrodescendência [online]. Salvador: EDUFBA, 2009. Newton Sucupira, a PósGraduação e a universidade. pp. 143-153.

BRAVO, R. S. Técnicas de investigação social: Teoria e exercícios.7 ed. Ver. Madrid: Paraninfo, 1991. 
FREIRE, P. Pedagogia da autonomia: saberes necessários à prática educativa. São Paulo: Editora Paz e Terra, 2014.

GIL, A. C. Como elaborar projetos de pesquisa. $4^{\text {a }}$ Ed. São Paulo: Atlas, 2002.

GIL, A. C. Métodos e técnicas da pesquisa social. $6^{\text {a }}$ Ed. São Paulo: Atlas, 2008.

IMBERNÓN, F. Formação continuada de professores. Porto Alegre: Artmed, 2010.

MAGALHÃES, R. C. B. P.; RAFFIN, F. N.; GUTIERRE L. S.; AZEVEDO, A. F. Formação docente na Pós-Graduação stricto sensu: experiências na Universidade Federal do Rio Grande do Norte. RBPG, Brasília, v.13, n. 31, p. 559 - 582, maio/ago. 2016.

MASOLA, W. J. ALLEVATO, N. S. G. Dificuldades de aprendizagem matemática de alunos ingressantes na educação superior. REBES - Rev. Brasileira de Ensino Superior, 2(1): 64-74, jan.-mar. 2016 - ISSN 2447-3944

NASSER, L. Ajudando a superar obstáculos na aprendizagem de cálculo. IM/UFRJ eCETIQT/SENAI, 2009.

OLIVEIRA, M. M. Como fazer pesquisa qualitativa. Petrópolis, Vozes, 2007.

PÁDUA, E. M. M. Metodologia da pesquisa: abordagem teórico-prática. Campinas: Papiros, 1997.

PIMENTA, S. G.; LIMA, M. S. L. Estágio e docência: diferentes concepções. Revista

Poíesis, Catalão, v. 3, n. 3 e 4, p. 5-24, 2006.

UFRN. Programa de Pós-Graduação em Saúde Coletiva. Apresentação. Natal, RN: (C) 2006-2019. Disponível em:

https://sigaa.ufrn.br/sigaa/public/programa/apresentacao.jsf?lc=pt_BR\&id=5670.

Acesso em: 19 de dezembro de 2018.

SÁ-SILVA, J. R.ALMEIDA, C. D. GUINDANI, J.F.Pesquisa documental: pistas teóricas e metodológicas. Revista Brasileira de História \& Ciências Sociais Ano I Número I - Julho de 2009.

SILVA, A. P.C. NASCIMENTO, E. F. VIEIRA, A. R. L. Cálculo diferencial e integral: obstáculos e dificuldades didáticas de aprendizagem. Caminhos da Educação

Matemática em Revista/Online, v. 7, n. 2, 2017.

SILVA, L. R. C.DAMACENO, A. D.MARTINS, M. C.R.SOBRAL, K. M. FARIAS, I. M. S. Pesquisa Documental: Alternativa Investigativa Na Formação Docente. IX congresso Nacional de Educação - EDUCERE. III Encontro Sul Brasileiro de Psicopedagogia 26 a 29 de outubro de 2009 - PUCPR. 
Tiely Virgíio da Hora Lima, Liliane dos Santos Gutierre

Estágio Docência: dificuldades encontradas pelos estagiários na disciplina de Cálculo I

UFRN. Resolução no 063/2010 - CONSEPE. Estabelece normas e regulamenta as atividades de Assistência à Docência na Graduação da Universidade Federal do Rio Grande do Norte. 2010. Disponível em:

http://www.ppged.ufrn.br/arquivos/resolucoes/resol_063_2010_consepe.pdf. Acesso em: 15 de setembro. 2018 . 